\title{
New Carbon - Carbon Coupling Reactions Mediated by Aluminum, Chromium and Samarium Reagents
}

\author{
Sorin I. Rosca \\ "Politehnica" University Bucharest, Romania \\ "C.D.Nenitescu" Organic Chemistry Department
}

\begin{abstract}
The aim of the research, reported in this presentation, is to exploit exceptional behaviour of the $\eta^{6}$-arenetricarbonylchromium complexes to stabilize practically all types of transient intermediates (benzyl carbenium ions, carbanions or radical type species). On this research line we have reported a facile and efficient coupling of $\eta^{6}$-complexed benzyl carbenium ions (generated by acid catalysis from corresponding alcohols/acetates) with highly nucleophilic arenes. The scope of this new carbon - carbon coupling reaction is enlarged by demonstrating its stereo specificity (retention of configuration). When this property is associated with the use of difunctional reagents (for both chromium complex and arene) the polycoupling process provides macromolecular products exhibiting totally controlled configuration. On the other hand, a new enantioselective synthesis of pertinent $\alpha$-amino acids based on stereo selective alkylation of an aza-allylic carbanion (generated by deprotonation of a planar chiral $\eta^{6}$-complexed Schiff base) is also reported.

Another route to generate new aryl - alkyl bonds consists in coupling of $\eta^{6}$ - (halogenobenzene) tricarbonylchromium complexes with organic halides or dialkyl ketones mediated by $\mathrm{SmI}_{2}$ /THF-HMPA. In this case, the high reaction rate and the selectivity of the carbon carbon coupling are attributed to an important labilization of the carbon - halogen bond as well as to the increase of radical - acceptor properties of the chromium complexes. Substantial experimental data are rationalized in terms of a reaction mechanism involving a Sm(IV) unstable species as transient intermediate. During our investigation on the reduction of organic halides with $\mathrm{Ni}$ - $\mathrm{Al}$ alloy, in aqueous alkaline solution, a new carbon - carbon coupling reaction of benzyl halides was discovered, providing a surprising, but definite existence of an organoaluminum compound in a protic solvent.
\end{abstract}

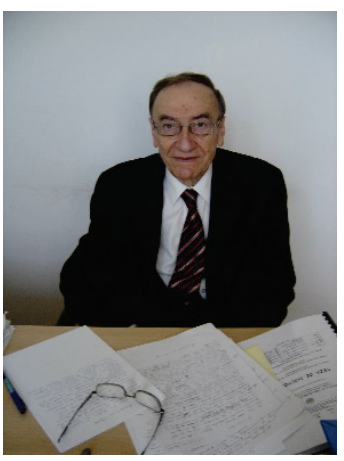

Biographical Sketch: Dr. Sorin ROSCA is a Professor of Organic Chemistry, Emeritus of "Politehnica" University Bucharest and is the President of Romanian Chemical Society. He holds a PhD in Organic Chemistry from Polytechnic Institute of Bucharest and a Dr. Honoris Causa title from Ovidius University, Constanta (Romania). His academic career in the Department on Organic Chemistry, Politehnica University includes, in different periods, positions of Head of the Department, Dean of the Faculty and Vice Rector of the University. His research interests concern organometallic chemistry, enantioselective reactions, mechanisms of organic and electoorganic reactions, authentications of food products by modern physical methods. He was Visiting Professor at "Paul Sabatier" University Toulouse (France), and Kyu-shu University Fukouka (Japan) and has numerous scientific presentations in universities from USA, UK, Japan, France, Germany, Greece, Turkey, etc. Prof. Rosca is a member of Romanian Academy of Technical Sciences and a corresponding member of the Academy of Sciences from Toulouse. He was co-director of $\mathrm{PhD}$ theses in the universities of Paris Sud, Orleans, Caen and Toulouse. His awards include Romanian ("Ordinul Muncii" si "Serviciu Credincios") and French ("Palmes Academiques") scientific decorations, Opera Omnia Award of the Politehnica University, the Prize of the chemical journal "Revista de Chimie" and others. 